DOI: https://doi.org/10.14311/TPFM.2022.022

\title{
NUMERICAL ASSESSMENT OF STRATIFICATION INFLUENCE IN SIMPLE ALGEBRAIC TURBULENCE MODEL
}

\author{
V. Uhlîr ${ }^{1}$, T. Bodnár ${ }^{1,2}$, M. Caggio ${ }^{2}$ \\ ${ }^{1}$ Faculty of Mechanical Engineering, Czech Technical University in Prague, \\ Karlovo Náměstí 13, 12135 Prague 2, Czech Republic. \\ 2 Institute of Mathematics, Czech Academy of Sciences, \\ Žitná 25, 11567 Prague 1, Czech Republic.
}

\begin{abstract}
This paper presents first few results obtained using a newly developed test code aimed at validation and cross-comparison of turbulence models to be applied in environmental flows. A simple code based on finite difference discretization is constructed to solve steady flows of incompresible non-homogeneous (variable denstity) fluids. For the first tests a simple algebraic turbulence model was implemented, containing stability function depending on the stratification via the gradient Richardson number. Numerical tests were performed in order to explore the capabilities of the new code and to get some insight into its behavior under different stratification. The two-dimensional simulations were performed using immersed boundary method for the flow over low smooth hill. The resulting flow fields are compared for selected Richarson numbers ranging from stable up to unstable stratification conditions.
\end{abstract}

Keywords: stratification, finite difference, immersed boundary, algebraic turbulence model, artificial compressibility method

\section{Introduction}

Many of the fluid flows of practical interest behave like incompressible, but with variable density. This is typical for environmental flows either because of mixing of different fluids or due to heating effects and associated thermal dilatation. By the action of (vertical) gravity force, the resulting density distribution often exhibits an important vertical variation. This leads to important buoyancy effects that affect both, the main flow as well as the turbulent fluctuations. For the main flow, the mathematical model has to contain the corresponding buoyancy forcing term. Typically the full non-homogeneous incompressible Navier-Stokes equations can be used, but more often the Boussinesq approximation is employed for simplicity (see Section 2 for details).

More complicated and less obvious is the impact of the fluid variable density and stratification on the turbulent fluctuations and their modelling. In general the negative vertical density gradient (stable stratification) helps to stabilize the flow and suppress the turbulent fluctuations. On the other hand the unstable stratification, when the vertical density gradient is positive, enhances the flow disturbances and turbulent fluctuations. This should be reasonably represented by the turbulence parametrisations used to describe this kind of flows.

The model used here is based on a simple algebraic turbulence model by Blackadar [1] generalized for stratified flows by Estoque and Bhumralkar [5]. Other models and detailed explanations about the variable density turbulence can be found for example in the book by Chassaing, Antonia, Anselmet, Joly, and Sarkar [3].

The aim of this work is to present a simple numerical code that was used to implement and test the algebraic turbulence model presented above in order to describe variable density incompressible fluid flow under stable, neutral and unstable stratification.

\section{Mathematical model}

In this work, we start from a non-homogenous incompressible model. The model consists of equations describing the conservation laws of mass and linear momentum. In particular, we consider 
the following system:

$$
\begin{aligned}
\nabla \cdot \widetilde{\boldsymbol{u}} & =0 \\
\frac{\partial \widetilde{\rho}}{\partial t}+\nabla \cdot(\widetilde{\rho} \widetilde{\boldsymbol{u}}) & =\nabla \cdot(\kappa \nabla \widetilde{\rho}) \\
\left(\frac{\partial \widetilde{\boldsymbol{u}}}{\partial t}+(\widetilde{\boldsymbol{u}} \cdot \nabla) \widetilde{\boldsymbol{u}}\right) & =-\frac{\nabla \widetilde{p}}{\widetilde{\rho}}+\nabla \cdot(\nu \nabla \widetilde{\boldsymbol{u}})+\boldsymbol{g} .
\end{aligned}
$$

Here, $\widetilde{\rho}(\boldsymbol{x}, t), \widetilde{\boldsymbol{u}}(\boldsymbol{x}, t)$ and $\widetilde{p}(\boldsymbol{x}, t)$ are the density, the velocity and the pressure of the fluid, respectively; $\kappa$ the thermal diffusion coefficient, $\nu$ the laminar kinematic viscosity and $\boldsymbol{g}=(0,0,-g)$ the gravity acceleration.

Now, we consider the Boussinesq approximation (see, for example, Spiegel and Veronis [12], Zeytounian [16]) and we decompose the density and pressure fields into a time-independent background state and its perturbation

$$
\begin{aligned}
& \tilde{\rho}(\boldsymbol{x}, t)=\rho_{0}(\boldsymbol{x})+\widetilde{\rho}^{\prime}(\boldsymbol{x}, t), \\
& \widetilde{p}(\boldsymbol{x}, t)=p_{0}(\boldsymbol{x})+\widetilde{p}^{\prime}(\boldsymbol{x}, t),
\end{aligned}
$$

where the density and the pressure background states are linked through the hydrostatic balance relation

$$
\nabla p_{0}=\rho_{0} \boldsymbol{g}
$$

Assuming that the density perturbations are small compared to the background field, namely $\widetilde{\rho}^{\prime} \ll \rho_{0}$, and using the decomposition (4) and (5) together with (6), after some algebra is possible to derive the following expression (for further details, see, for example, Wyngaard [15])

$$
-\frac{\nabla \widetilde{p}}{\widetilde{\rho}}+\boldsymbol{g} \approx-\frac{1}{\rho_{0}} \nabla \widetilde{p}^{\prime}+\frac{\widetilde{\rho}^{\prime}}{\rho_{0}} \boldsymbol{g} .
$$

Consequently, the system (1) - (3) could be rewritten as follows

$$
\begin{aligned}
\nabla \cdot \widetilde{\boldsymbol{u}} & =0, \\
\frac{\partial \widetilde{\rho}^{\prime}}{\partial t}+\widetilde{\boldsymbol{u}} \cdot \nabla \widetilde{\rho}^{\prime} & =\nabla \cdot\left(\kappa \nabla \widetilde{\rho}^{\prime}\right)-\widetilde{\boldsymbol{u}} \cdot \nabla \rho_{0}, \\
\left(\frac{\partial \widetilde{\boldsymbol{u}}}{\partial t}+(\widetilde{\boldsymbol{u}} \cdot \nabla) \widetilde{\boldsymbol{u}}\right) & =-\frac{1}{\rho_{0}} \nabla \widetilde{p}^{\prime}+\nabla \cdot(\nu \nabla \widetilde{\boldsymbol{u}})+\frac{\widetilde{\rho}^{\prime}}{\rho_{0}} \boldsymbol{g},
\end{aligned}
$$

where we assumed that the background density $\rho_{0}$ is a linear function of the space coordinates.

Now, following Reynolds [11], we decompose $\widetilde{\rho}^{\prime}, \widetilde{\boldsymbol{u}}$ and $\widetilde{p}^{\prime}$ into a mean flow component, or average, and a turbulent fluctuation, namely

$$
\widetilde{\rho}^{\prime}=\overline{\rho^{\prime}}+\rho^{\prime}, \quad \widetilde{\boldsymbol{u}}=\overline{\boldsymbol{u}}+\boldsymbol{u}, \quad \widetilde{p}^{\prime}=\overline{p^{\prime}}+p^{\prime},
$$

where $\bar{\rho}^{\prime}, \overline{\boldsymbol{u}}$ and $\overline{p^{\prime}}$ represent the mean quantities, and $\rho^{\prime}, \boldsymbol{u}$ and $p^{\prime}$ the turbulent components. Plugging the decomposition (11) into (8), (9) and (10), averaging the equations, and noting that the average of the turbulent fluctuations is zero, we end up with the following system (for a detailed derivation see, for example, Garratt [7]):

$$
\begin{aligned}
\nabla \cdot \overline{\boldsymbol{u}} & =0 \\
\frac{\partial \overline{\rho^{\prime}}}{\partial t}+\overline{\boldsymbol{u}} \cdot \nabla \overline{\rho^{\prime}} & =\nabla \cdot\left(\kappa \nabla \overline{\rho^{\prime}}\right)-\nabla \cdot \boldsymbol{h}-\overline{\boldsymbol{u}} \cdot \nabla \rho_{0} \\
\left(\frac{\partial \overline{\boldsymbol{u}}}{\partial t}+(\overline{\boldsymbol{u}} \cdot \nabla) \overline{\boldsymbol{u}}\right) & =-\frac{\nabla \overline{p^{\prime}}}{\rho_{0}}+\nabla \cdot(\nu \nabla \overline{\boldsymbol{u}})-\nabla \cdot \boldsymbol{\tau}+\frac{\overline{\rho^{\prime}}}{\rho_{0}} \boldsymbol{g},
\end{aligned}
$$

where $h_{j}=\overline{\rho^{\prime} u_{j}}$ and $\tau_{i j}=\overline{u_{i} u_{j}}$ are turbulent mass and momentum fluxes, respectively. In order "to close" the system (12) - (14), we assume that the turbulent fluxes can be written in terms of the flux-gradient approximation (see Boussinesq [2]), namely

$$
\boldsymbol{\tau}=-\nu_{T} \nabla \overline{\boldsymbol{u}}, \quad \boldsymbol{h}=-\kappa_{T} \nabla \bar{\rho},
$$


where the turbulent viscosity $\nu_{T}$ and the turbulent diffusivity $\kappa_{T}$ are quantities to be determined, for example, as functions of the average fields. This approximation implies the existence of a diffusive process, or at least that the turbulent fluxes can be approximated by such a process (see, for example, Tampieri [13]). Consequently, we end up with the following system of equations

$$
\begin{aligned}
\nabla \cdot \overline{\boldsymbol{u}} & =0 \\
\frac{\partial \overline{\rho^{\prime}}}{\partial t}+\overline{\boldsymbol{u}} \cdot \nabla \overline{\rho^{\prime}} & =\nabla \cdot\left(\left(\kappa+\kappa_{T}\right) \nabla \overline{\rho^{\prime}}\right)+\overline{\boldsymbol{u}} \cdot \gamma \\
\frac{\partial \overline{\boldsymbol{u}}}{\partial t}+(\overline{\boldsymbol{u}} \cdot \nabla) \overline{\boldsymbol{u}} & =-\frac{\nabla \overline{p^{\prime}}}{\rho_{0}}+\nabla \cdot\left(\left(\nu+\nu_{T}\right) \nabla \overline{\boldsymbol{u}}\right)+\frac{\overline{\rho^{\prime}}}{\rho_{0}} \boldsymbol{g}
\end{aligned}
$$

where $\gamma=(0,0, \gamma)$, with $\gamma=-\frac{\partial \rho_{0}}{\partial z}$ is a vector parallel to the gravity acceleration.

In order to determine the turbulent viscosity $\nu_{T}$ and the turbulent thermal diffusivity $\kappa_{T}$, a mixing length model is introduced (see Prandtl [10]). The idea is based on the assumption that a parcel of fluid is keeping its original momentum for a characteristic length $\ell$ before mixing with the surrounding fluid. A relation between the turbulent viscosity and the mixing length $\ell$ has been originally proposed by Blackadar [1](written here for two-dimensional flows)

$$
\nu_{T}=\ell^{2}\left[\left(\frac{\partial u}{\partial z}\right)^{2}+\left(\frac{\partial w}{\partial z}\right)^{2}\right]^{1 / 2}, \quad \text { with mixing length } \quad \ell=\frac{\kappa z}{1+\frac{\kappa z}{\ell_{\infty}}}
$$

where $z$ is the distance from the ground, $\kappa=0.41$ is von Kármán's constant and $\ell_{\infty}$ is a suitable asymptotic value such that $\ell \approx \ell_{\infty}$ at large distances from the ground. In our setting, $\ell_{\infty}=H / 2$.

In order to take into account the effects of the stratification, the above model was generalized by Estoque and Bhumralkar [5] introducing a stability function $\mathcal{G}$ such that

$$
\begin{aligned}
\nu_{T}=\ell^{2}\left[\left(\frac{\partial u}{\partial z}\right)^{2}+\left(\frac{\partial w}{\partial z}\right)^{2}\right]^{1 / 2} \cdot \mathcal{G}, & \text { where } \\
\mathcal{G}=(1+3 R i)^{-2}, & \text { for } R i>0, \\
\mathcal{G}=(1-3 R i)^{2}, & \text { for } R i \leq 0 .
\end{aligned}
$$

The figure 1 shows the dependency of the stability function $\mathcal{G}$ on the Richardson number $R i$ with marking of the three specific values, used in simulations. Thus it is obvious, how the choice of $R i$ increases or lowers the magnitude of turbulent kinematic viscosity according to equation (12).

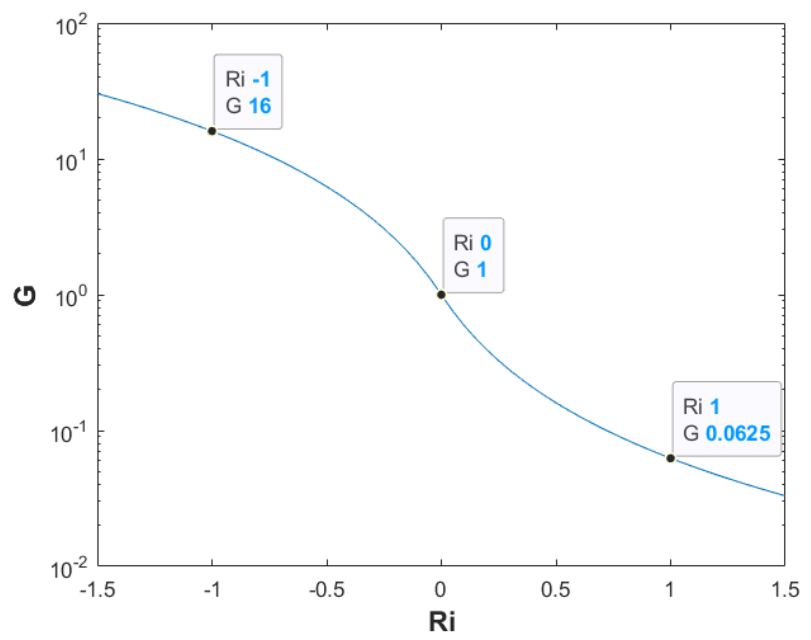

Figure 1: Graph of the stability function $\mathcal{G}$ in dependence on the Richardson number

Here $R i$ stands for the Richardson number, which is determined by

$$
R i=\frac{g}{\rho_{*}} \frac{\gamma}{\sum_{i=1}^{2}\left(\frac{\partial u_{i}}{\partial z}\right)^{2}} .
$$


Finally, the turbulent diffusivity is expressed through the turbulent Prandtl number $\operatorname{Pr}_{T}$ as

$$
\kappa_{T}=\nu_{T} / P r_{T},
$$

where $\operatorname{Pr}_{T}=0.74$ in our setting.

\section{$3 \quad$ Numerical methods}

Numerical simulations discussed in this paper correspond to two-dimensional steady case. The numerical methods used to obtain results for the above derived model are briefly described in the following few paragraphs. In order to simplify notation, the turbulent averaging overbars are dropped and starting from now the velocity, density and pressure fields are considered as Reynoldsaveraged when turbulent flow is considered. Also the prime is removed from $p^{\prime}$ and $p$ will denote the pressure perturbation in the sense (5) and (11).

\subsection{Artificial compressibility}

In order to be able to numerically solve above described equations, the artificial compressibility method is used. This method was first introduced in Chorin [4]. The advantage od this method is its simplicity and robustness. The basic idea is extension of the continuity equation by adding timederivative of pressure scaled by coefficient of artificial compressibility $\beta$. The continuity equation then takes the form:

$$
\frac{1}{\beta^{2}} \frac{\partial p}{\partial t}+\nabla \cdot \boldsymbol{u}=0
$$

The coefficient $\beta$ is called artificial speed of sound. This non-physical added time-derivative disappears as soon as the solution reaches its steady state and divergence-free velocity field is recovered.

\subsection{Numerical scheme}

The numerical simulations are conducted with use of finite difference method. Specifically, explicit MacCormack scheme is used. This scheme is one of many variants of Lax-Wendroff schemes and is based on two step (predictor-corrector) implementation. For brevity, the discretization is explained just for one of the variables, the horizontal velocity $u$. Applying the MacCormack scheme on the equation (14), leads to following predictor-corrector formulas:

Predictor:

$$
\begin{aligned}
u_{i, j}^{n+1 / 2} & =u_{i, j}^{n}-\Delta t\left[\frac{p_{i+1, j}^{n}-p_{i, j}^{n}}{\rho_{*} \Delta x}+u_{i+1 / 2, j}^{n} \frac{u_{i+1, j}^{n}-u_{i, j}^{n}}{\Delta x}+w_{i, j+1 / 2}^{n} \frac{u_{i, j+1}^{n}-u_{i, j}^{n}}{\Delta z}-\right. \\
& -\left(\frac{\nu_{i+1 / 2, j}^{n} \cdot\left(u_{i+1, j}^{n}-u_{i, j}^{n}\right)-\nu_{i-1 / 2, j}^{n} \cdot\left(u_{i, j}^{n}-u_{i-1, j}^{n}\right)}{\Delta x^{2}}+\right. \\
& \left.\left.+\frac{\nu_{i, j+1 / 2}^{n} \cdot\left(u_{i, j+1}^{n}-u_{i, j}^{n}\right)-\nu_{i, j-1 / 2}^{n} \cdot\left(u_{i, j}^{n}-u_{i, j-1}^{n}\right)}{\Delta z^{2}}\right)\right]
\end{aligned}
$$

$$
\begin{aligned}
& \widehat{u}_{i, j}^{n+1}=\frac{u_{i, j}^{n}+u_{i, j}^{n+1 / 2}}{2}-\frac{\Delta t}{2}\left[\frac{p_{i, j}^{n}-p_{i-1, j}^{n}}{\rho_{*} \Delta x}+u_{i-1 / 2, j}^{n+1 / 2} \frac{u_{i, j}^{n+1 / 2}-u_{i-1, j}^{n+1 / 2}}{\Delta x}+w_{i, j-1 / 2}^{n+1 / 2} \frac{u_{i, j}^{n+1 / 2}-u_{i, j-1}^{n+1 / 2}}{\Delta z}-\right. \\
& -\left(\frac{\nu_{i+1 / 2, j}^{n+1 / 2} \cdot\left(u_{i+1, j}^{n+1 / 2}-u_{i, j}^{n+1 / 2}\right)-\nu_{i-1 / 2, j}^{n+1 / 2} \cdot\left(u_{i, j}^{n+1 / 2}-u_{i-1, j}^{n+1 / 2}\right)}{\Delta x^{2}}+\right. \\
& \left.\left.+\frac{\nu_{i, j+1 / 2}^{n+1 / 2} \cdot\left(u_{i, j+1}^{n+1 / 2}-u_{i, j}^{n+1 / 2}\right)-\nu_{i, j-1 / 2}^{n+1 / 2} \cdot\left(u_{i, j}^{n+1 / 2}-u_{i, j-1}^{n+1 / 2}\right)}{\Delta z^{2}}\right)\right] \text {. }
\end{aligned}
$$

This numerical scheme is formally of second order of accuracy. For more details on MacCormack scheme see for example the classical books of Hirsch [8] or Fletcher [6] or the thesis Uhlír [14], where the method was used and validated for laminar flow.

The newly obtained value $\widehat{u}_{i, j}^{n+1}$ is not yet definitive and numerical stabilization is applied, which is described in the next paragraph. 


\subsection{Stabilization}

In order to suppress numerical oscillations in the solution, a fourth order artificial viscosity is implemented in the code, so the final value at the time level $n+1$ is obtained as

$$
u_{i, j}^{n+1}=\widehat{u}_{i, j}^{n+1}+D^{4} \widehat{u}_{i, j}^{n}
$$

where the fourth order numerical viscosity has the form $D^{4} \widehat{u}_{i, j}^{n}=D_{x}^{4} \widehat{u}_{i, j}^{n}+D_{z}^{4} \widehat{u}_{i, j}^{n}, \quad$ with :

$$
\begin{aligned}
& D_{x}^{4} \widehat{u}_{i, j}^{n}=\epsilon\left(\widehat{u}_{i-2, j}^{n}-4 \widehat{u}_{i-1, j}^{n}+6 \widehat{u}_{i, j}^{n}-4 \widehat{u}_{i+1, j}^{n}+\widehat{u}_{i+2, j}^{n}\right), \\
& D_{z}^{4} \widehat{u}_{i, j}^{n}=\epsilon\left(\widehat{u}_{i, j-2}^{n}-4 \widehat{u}_{i, j-1}^{n}+6 \widehat{u}_{i, j}^{n}-4 \widehat{u}_{i, j+1}^{n}+\widehat{u}_{i, j+2}^{n}\right),
\end{aligned}
$$

where $\epsilon$ is suitably chosen coefficient.

\section{Computational setup}

The considered problem was motivated by a stratified flow in the atmospheric boundary layer with an obstacle representing a hill. For simplicity the problem is only considered in two-dimensions. The geometry of the computational domain including the size and shape of the obstacle is shown in the Fig. 2.

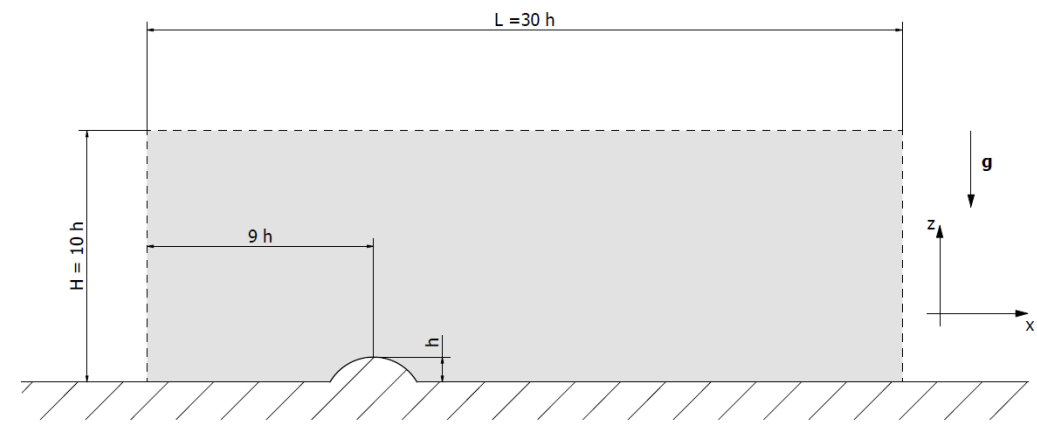

Figure 2: Computational domain

The gray region represents computational domain of length $L=3 \mathrm{~m}$ and height $H=1 \mathrm{~m}$ (laboratory scale water tank is considered). Hill is has circular shape with radius $r=0.2 \mathrm{~m}$ and $h=\frac{1}{10} H$. In simulations the hill is represented by simple immersed boundary method, so no special grid adjustment is needed.

The fluid considered in this study is water as it is typically used to study stratified flows over hills in laboratories (see e.g. Hunt and Snyder [9]). Therefore the laminar viscosity was set to $\nu=10^{-4} \mathrm{~m}^{2} \cdot \mathrm{s}^{-1}$ and density on the wall $\rho_{w}=1000 \mathrm{~kg} \cdot \mathrm{m}^{-3}$. Background density $\rho_{0}$ is then given by the linear profile $\rho_{0}(z)=\rho_{*}+\gamma z$, where $\gamma$ is determined from chosen Richardson number from the formula $(22)$, considering characteristic density $\rho_{*}$ and approximate velocity gradient $\frac{\partial u}{\partial z} \approx u_{\infty} / \frac{H}{2}$ where $u_{\infty}=1 m \cdot s^{-1}$.

\subsection{Boundary conditions}

Boundary conditions are set to emulate the flow in atmospheric boundary layer. The only physical boundary in this kind of flows is the solid impermeable wall on which the no-slip (i.e. homogeneous Dirichlet) boundary conditions are prescribed for velocity, while homogeneous Neumann condition is used for density perturbation.

On the inlet (left) boundary the horizontal velocity component is set the expected velocity profile (parabolic up to $z=H / 2$, extended by constant above this level), vertical velocity component and density perturbation is set $w=\rho^{\prime}=0$.

On the outlet (right) boundary there homogeneous Neumann conditions set for velocity components and density perturbation $\frac{\partial u}{\partial x}=\frac{\partial w}{\partial x}=\frac{\partial \rho^{\prime}}{\partial x}=0$ while pressure is extrapolated.

On the upper boundary is considered a free stream, thus for all the calculated quantities is homogeneous Neumann condition applied $\frac{\partial u}{\partial z}=\frac{\partial w}{\partial z}=\frac{\partial \rho^{\prime}}{\partial z}=\frac{\partial p}{\partial z}=0$. 


\section{$5 \quad$ Numerical results}

Some of the first results obtained using newly developed code are presented in this section. The focus is on qualitative comparison of flows under different stratification, simulated with or without the turbulence model. The character of the flow is mainly affected by stratification, which corresponds to Richardson number $R i$. In our simulations three different values or $R i$ were chosen equal to $R i=0, R i=1$ and $R i=-1$ corresponding to neutral, stable and unstable stratification respectively.

\subsection{Laminar case}

For reference and comparison, the first set of simulations was performed without the turbulence model, which corresponds to setting $\nu_{T}=0$, so only the kinematic (laminar) constant viscosity $\nu$ is considered. The results are presented in Fig. 3 in the form of streamlines. The standard case with neutral stratification is placed first, so it can be easily compared with the results shown below for the stable and unstable stratification.

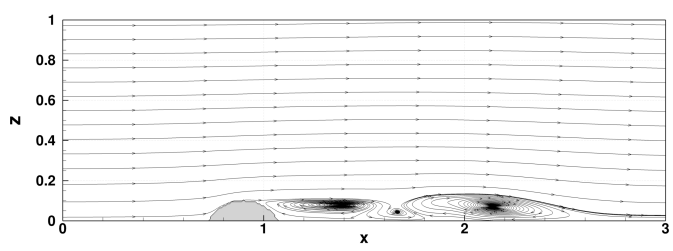

(a) Neutral stratification $R i=0.0$

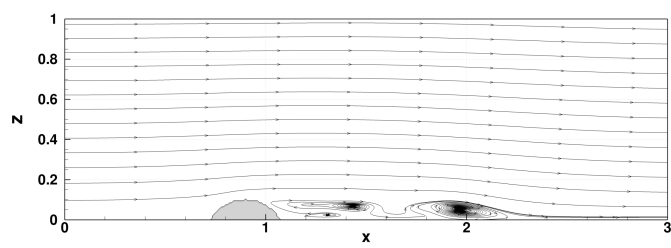

(b) Stable stratification $R i=1.0$

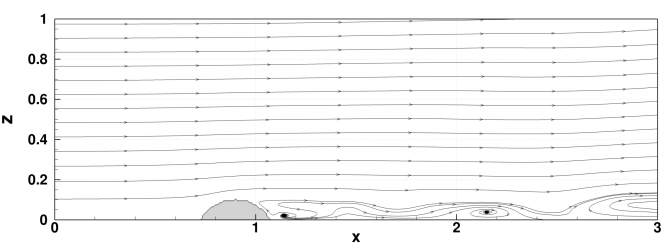

(c) Unstable stratification $R i=-1.0$

Figure 3: Streamlines - Laminar flow

Without the turbulence model, the viscosity is quite low, which results in massive separation behind the hill. The vortices are detaching from the lee side of the hill and traveling towards the outlet. For the stably stratified flow the vortices seem to be a bit smaller and stronger attached to the hill than in the unstable case. The resulting flow field is unsteady, so the Fig. 3 only shows an instantaneous snapshot of the solution. The viscosity is constant in this case, so the plots are omitted.

\subsection{Turbulent case}

In this set of tests the turbulence model was switched on, so the turbulent viscosity $\nu_{T}$ was determined from (19) and (20). In order to assess the effect of the stability function $\mathcal{G}$, all simulations were first run without the function $\mathcal{G}$ (i.e. by setting $\mathcal{G}=1.0$ ) and then the same simulations were performed with the function $\mathcal{G}$ determined from (21). In addition to streamlines, fields of total kinematic viscosity, containing both laminar part $\nu$ and turbulent eddy viscosity $\nu_{T}$, are presented for all simulations.

\section{Without the stability function $\mathcal{G}$}

In case only the standard model of turbulence is used, without the stability function $\mathcal{G}$, the flow field changes dramatically. The recirculation zones on the lee side of the hill are steady, strongly attached to the hill. The added turbulent viscosity helped to stabilize the flow and produce steady velocity fields. The size and shape of recirculation zone is very similar for all three levels of stratification, with only minor changes further from the hill. 


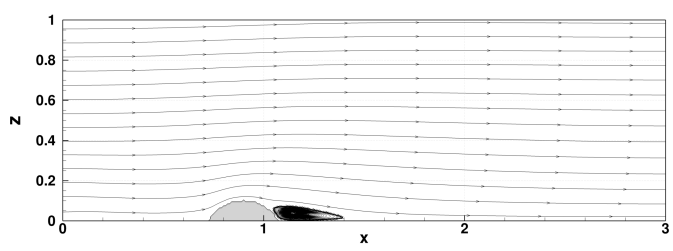

(a) Neutral stratification $R i=0.0$

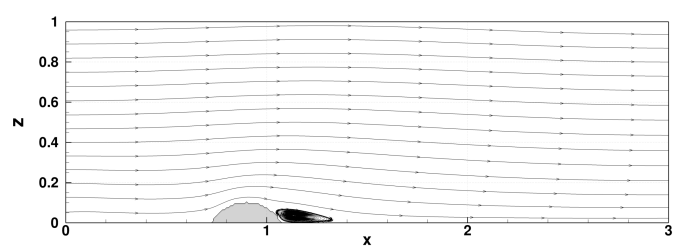

(b) Stable stratification $R i=1.0$

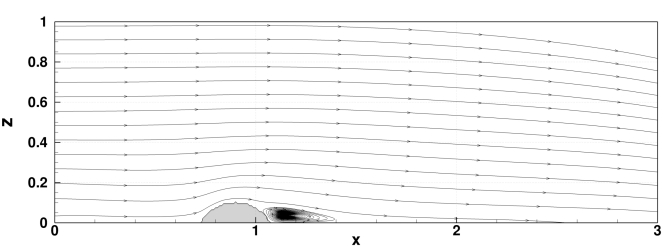

(c) Unstable stratification $R i=-1.0$

Figure 4: Streamlines - Turbulent flow - without the stability function $\mathcal{G}$

The contours of total viscosity $\left(\nu+\nu_{T}\right)$ are shown in Fig. 5 . There is a visible increase in the viscosity value in the layer just above the hill. Turbulent viscosity is (as expected) highest in the case of unstable stratification, however the values are still of the same order as in the neutral and stable case. It seems that similar values of the turbulent viscosity in all cases lead to similar smoothing effects in the flow field, resulting in only small differences between the flow fields for the three levels of stratification.

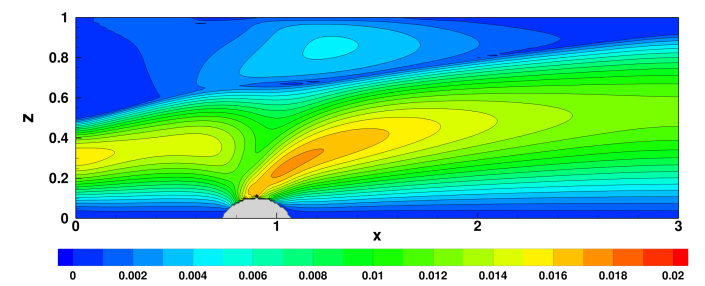

(a) Neutral stratification $R i=0.0$

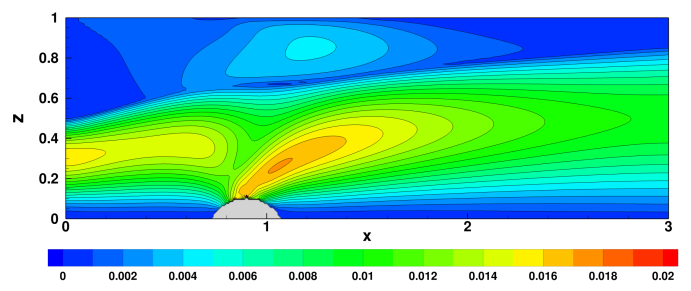

(b) Stable stratification $R i=1.0$

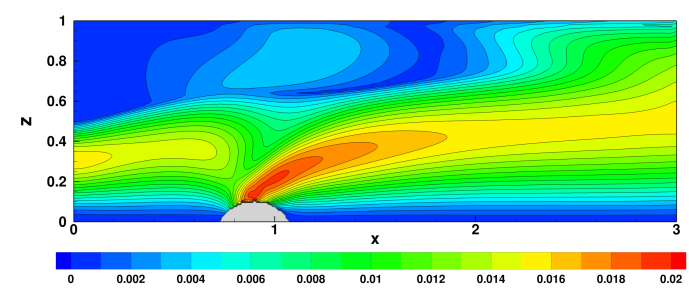

(c) Unstable stratification $R i=-1.0$

Figure 5: Turbulent viscosity - without the stability function $\mathcal{G}$

\section{With the stability function $\mathcal{G}$}

Simulations with the stability function defined by (21) give significantly different results in comparison to the previous cases. The main reason is evidently due to stability function $\mathcal{G}$ that is used as a constant multiplicative factor for the whole turbulent viscosity field. As it can be seen from Fig. 2, while for the neutral stratification $\mathcal{G}=1.0$, for the stable case with $R i=1$ it reduces to $\mathcal{G}=1 / 16$, while in the unstable case with $R i=-1$ it jumps to $\mathcal{G}=16$. This leads to huge differences in the magnitude of the turbulent viscosity, that significantly affects the flow. It is interesting that the unsteady stratification significantly reduced the size of the recirculation zone, which can possibly be caused by the increase of the momentum transfer to the boundary layer due to contribution of turbulent viscosity. On the other hand the largest recirculation appears in the case of stable stratification where the contribution of turbulent viscosity is smallest. 


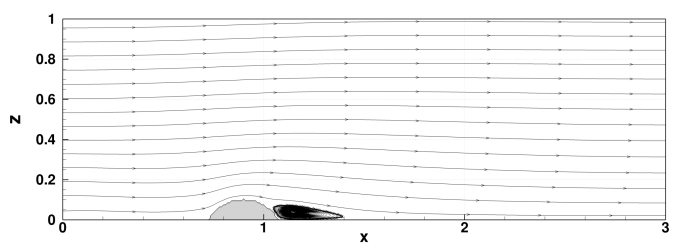

(a) Neutral stratification $R i=0.0$

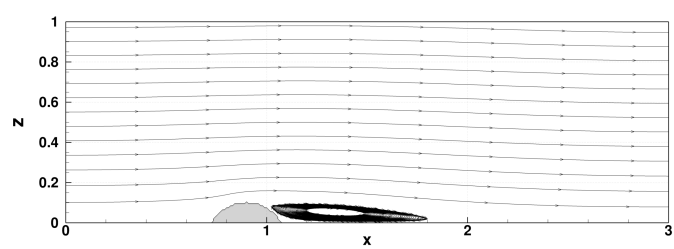

(b) Stable stratification $R i=1.0$

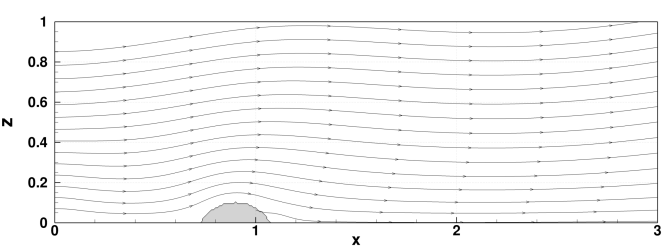

(c) Unstable stratification $R i=-1.0$

Figure 6: Streamlines - Turbulent flow - with the stability function $\mathcal{G}$

The use of stability function $\mathcal{G}$ significantly affects the turbulent viscosity fields as it can be seen in Fig. 7 (note different color scale used for each picture). As expected, the turbulent viscosity for the unstable stratification is much higher than in the stable case.

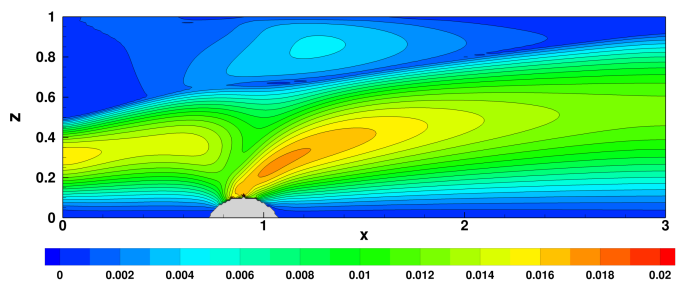

(a) Neutral stratification $R i=0.0$

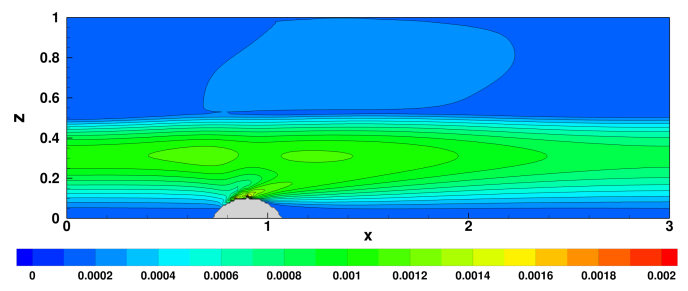

(b) Stable stratification $R i=1.0$



(c) Unstable stratification $R i=-1.0$

Figure 7: Turbulent viscosity - with the stability function $\mathcal{G}$

\section{Discussions and Conclusions}

Some of the first results obtained using our new code were presented. Although the code is rather simple and so is the algebraic model of turbulence used in it, the results already showed some interesting features and trends.

- For the chosen Reynolds number the numerical simulations seem to be stable, providing results without visible numerical oscillations.

- The use of the stability function $\mathcal{G}$ strongly affects the results for both stable and unstable stratification. This effect should further be verified for some more realistic flow conditions, closer to typical wind flow in the atmosphere.

- Some kind of validation of the turbulence model should be provided in order to be able to assess the uncertainty introduced by its use.

- More advanced turbulence models should be implemented and tested to see how much the results will differ from what was obtained using the very simple model used in this study. 


\section{Acknowledgment}

The financial support for the present work was provided by the Czech Science Foundation under the grant No. P201-19-04243S and by the Research Plan RVO 67985840. .

\section{References}

[1] A.K. Blackadar: The vertical distribution of wind and turbulent exchange in a neutral atmosphere. Journal of Geophysical Research, 67(8):3095-3102, 1962.

[2] J. Boussinesq: Essai sur la théorie des eaux courantes, volume Tome XXIII of Memoires presentes par divers savants a l? Academie des Sciences de l?Institut National de France. Imprimerie Nationale, Paris., 1877.

[3] P. Chassaing, R.A. Antonia, F. Anselmet, L. Joly, and S. Sarkar: Variable Density Fluid Turbulence. Springer, 2002.

[4] A.J. Chorin: A numerical method for solving incompressible viscous flows problems. Journal of Computational Physics, 2(1):12-26, 1967.

[5] M.A. Estoque and C.M. Bhumralkar: Flow over a localized heat source. Monthly Weather Review, 97(12):850-859, 1969.

[6] C. A. J. Fletcher: Computational Fluid Dynamics - The Basics with Applications. McGrawHill, 1995.

[7] J. R. Garratt: The atmospheric boundary layer. Cambridge atmospheric and space series. Cambridge University Press, 1992.

[8] C. Hirsch: Numerical computation of internal and external flows, volume 1,2. John Willey \& Sons, 1988.

[9] J. C. R. Hunt and W. H. Snyder: Experiments on stably and neutrally stratified flow over a model three-dimensional hill. Journal of Fluid Mechanics, 96(4):671-704, 1980.

[10] L. Prandtl: The mechanics of viscous fluids. In W. F. Durand, editor, Aerodynamic Theory, volume III., chapter G., pages 35-208. Julius Springer, 1935.

[11] O. Reynolds: On the dynamical theory of incompressible viscous fluids and the determination of the criterion. Philosophical Transactions of the Royal Society of London. (A.), 186:123-164, 1895.

[12] E.A. Spiegel and G. Veronis: On the boussinesq approximation for a compressible fluid. Astrophysical Journal, 131:442-447, 1960.

[13] F. Tampieri: Turbulence and Dispersion in the Planetary Boundary Layer. Springer, 2017.

[14] V. Uhliŕ: Numerical solution of stratified fluids flows. Master's thesis, Czech Technical University in Prague, 2021. (in Czech).

[15] J.C. Wyngaard: Turbulence in the Atmosphere. Cambridge University Press, 2010.

[16] R.K. Zeytounian: Joseph boussinesq and his approximation: a contemporary view. Comptes Rendus Mécanique, 331(8):575-586, 2003. 